\title{
The Periconceptional Healthy Dietary Pattern Mitigates Estradiol Response and Increases the Chance of Pregnancy after Conventional IVF/ICSI Treatment
}

\author{
Twigt JM1, Vujkovic M11, H de Vries J4 , Lindemans J2, Laven JSE ${ }^{1,3}$ \\ and Steegers Theunissen RPM1* \\ ${ }^{1}$ Obstetrics and Gynecology, Netherlands \\ ${ }^{2}$ Clinical Chemistry, Netherlands \\ ${ }^{3}$ Division of Reproductive Medicine, Erasmus MC, University Medical Centre \\ Rotterdam, Netherlands \\ ${ }^{4}$ Human Nutrition, Wageningen University, Netherlands
}

\begin{tabular}{|c|}
\hline Research Article \\
Volume 2 Issue 1 \\
Received Date: February 22, 2018 \\
Published Date: April 16, 2018 \\
DoI: 10.23880 /whsj-16000110 \\
\hline
\end{tabular}

*Corresponding author: Régine PM Steegers-Theunissen, Professor in Periconception Epidemiology, Department of Obstetrics and Gynaecology Erasmus MC, University Medical Centre Rotterdam, Erasmus MC, PO Box 2040, 3000CA, Rotterdam, Netherlands, Tel: +31 10 7032601; Fax: +31 10 7036815; Email: r.steegers@erasmusmc.n

\section{Abstract}

Background: Subfertility rates are increasing worldwide. Nutrition influences natural fertility, but also after assisted reproduction. In addition, the ovarian response after ovarian stimulation treatment possibly influences assisted reproduction treatment outcome.

Aim: to investigate the impact of periconceptional nutrition on endocrine response and reproductive outcome after controlled ovarian hyperstimulation and In Vitro Fertilization treatment (IVF) with and without Intracytoplasmatic Sperm Injection (ICSI).

Methods: We assessed the habitual dietary intake in 203 women undergoing IVF, using a Food Frequency Questionnaire and performed Principal Components Analysis to identify dietary patterns. Blood samples taken at CD2 preceding treatment and on the day of hCG were used for determination of endocrine and one-carbon metabolism biomarkers. The relation between the identified dietary patterns and estradiol $\left(\mathrm{E}_{2}\right)$ response after $\mathrm{COH}$ and pregnancy chance was assessed using regression analysis.

Results: Independent of stimulation protocol, adherence to a Healthy dietary pattern, characterized by high intakes of fruits, whole grains and margarine, associates with lower $\mathrm{E}_{2}$ concentrations on the day of hCG. There was no difference in baseline anthropomorphic parameters between the groups of adherence. After adjustments, every one-unit increase in 


\section{Women's Health Science Journal}

adherence to this dietary pattern decreased the $\mathrm{E}_{2}$ response by $16.4 \%$. Similarly, adherence to the Healthy dietary pattern associates with a $61 \%$ increased chance of ongoing pregnancy after IVF/ICSI treatment.

Conclusion: Adherence to a habitual Healthy dietary pattern mitigates the $E_{2}$ response and increases the chance of ongoing pregnancy after IVF/ICSI treatment. This data contributes to the explanation of the beneficial effects of a healthy lifestyle on IVF/ICSI treatment outcomes and provides an opportunity to improve the quality as well as the further personalization of IVF/ICSI treatment.

Keywords: Nutrtion; Assisted Reproduction; Folic acid

Abbreviations: IVF: In Vitro Fertilization; ICSI: Intracytoplasmic Sperm Injection; CD: Cycle Day; HCG: Human Chorionic Gonadotropin; COH: Controlled Ovarian Hyperstimulation; FFQ: Food Frequency Questionnaire; PCA: Principal Components Analysis; FSH: Follicle Stimulating Hormone; AMH: Anti-mullerian Hormone; RBC: Red Blood Cell; PLP: Pyridoxial-5-Phosphate.

\section{Introduction}

In Vitro Fertilization and Intracytoplasmic Sperm Injection (IVF/ICSI) are marginally successful techniques to achieve pregnancy in subfertile couples [1]. The propensity for IVF/ICSI treatment success is also determined by a sufficient ovarian response to Controlled Ovarian Hyperstimulation $(\mathrm{COH})$, i.e. the number of growing ovarian follicles and the quantity of steroid hormone production [2]. If one can identify modifiable factors that affect the ovarian response to $\mathrm{COH}$ as well as the chance of ongoing pregnancy, new opportunities become available to improve and further personalize IVF/ICSI treatment.

$\mathrm{COH}$ and supraphysiological estradiol $\left(\mathrm{E}_{2}\right)$ levels observed during $\mathrm{COH}$ cycles detrimentally affect endometrial development [3-5]. Therefore, during traditional IVF/ICSI treatment, the embryo is possibly transferred to a relatively unreceptive endometrium [5]. There has been much debate about the relevance of the ovarian response on clinical outcomes, such as ongoing pregnancy [6-9]. It could be helpful to not just focus on the influence of the ovarian response on IVF/ICSI treatment outcome through the effects of steroid hormone production on the endometrium, but to consider the influence of the ovarian response (of which $E_{2}$ is a marker) on normal oocyte and follicular development [10-12].
Demographic, ultrasound and endocrine markers can predict the ovarian response to $\mathrm{COH}$, and provide opportunities for a more personalized treatment [2]. In addition, there are various treatments to control the ovarian response to $\mathrm{COH}$. Nevertheless, there is still residual variability in the ovarian response that cannot be explained by such factors, where possibly maternal environmental factors also play a role. Unfortunately, except for overweight, such factors comprise nonmodifiable factors, with little variability between treatment cycles and are therefore not suitable to modify the ovarian response to $\mathrm{COH}[13,14]$. Modifiable factors, such as diet and other lifestyle factors could help control and modify the ovarian response to $\mathrm{COH}$ within the context of a woman's demographic, ultrasound and endocrine constitution.

In previous studies, we and others showed that periconceptional folic acid supplement use mitigates the $\mathrm{E}_{2}$ response to $\mathrm{COH}$ and improves embryo quality. Furthermore, the periconceptional diet and lifestyle associate with the subsequent chance of pregnancy after IVF/ICSI treatment, but also with the risk of subfertility and natural fecundity [15-19]. The underlying mechanisms are largely unknown.

Within this body of evidence the current study aims to identify a habitual diet of women undergoing IVF/ICSI treatment using two different $\mathrm{COH}$ protocols that influences the $\mathrm{E}_{2}$ response to $\mathrm{COH}$ and clinical reproductive outcomes. In future, the results may contribute to improve the quality as well as the further personalization of subfertility treatment, in particular IVF/ICSI treatment.

\section{Materials and Methods}

The Food, Lifestyle and Fertility Outcome study was designed as a periconceptional cohort to investigate the 


\section{Women's Health Science Journal}

relationship between nutrition, lifestyle and IVF/ICSI treatment outcomes in which a randomized controlled trial was embedded to study a mild and conventional $\mathrm{COH}$ protocol. This study has been described in detail before [17-20]. Participation in the RCT was based on the patient's willingness to undergo randomization to either a mild or conventional stimulation protocol or additionally, a BMI $>29 \mathrm{~kg} / \mathrm{m}^{2}$ precluded participation in the randomized controlled trial, as opposed to $>37 \mathrm{~kg} / \mathrm{m}^{2}$ in the observational study.

After inclusion and before treatment commenced participants received a self-administered questionnaire addressing age, educational level, medical history, BMI, ethnicity, medication use, smoking, and use of folic acid and other vitamin supplements. In addition, all participants completed a Food Frequency Questionnaire (FFQ) to estimate food intake covering the previous 4 weeks. The FFQ was developed by the division of Human Nutrition, Wageningen University, and validated for intake of energy, B-vitamins, and mono- and polyunsaturated fatty acids [21]. The FFQ was provided on the day of oocyte retrieval or semen sample collection and returned on the day of embryo transfer.

The Dutch Central Committee for Human Research and the medical ethical and institutional review board of the Erasmus MC, University Medical Centre in Rotterdam approved the study protocol. Participants provided written informed consent before participation and the obtained materials and questionnaires were processed anonymously.

\section{IVF/ICSI Procedure}

Patients received the conventional or mild $\mathrm{COH}$ treatment. Patients undergoing conventional $\mathrm{COH}$ were treated with the GnRH agonist Triptorelin (Decapeptyl ${ }^{\circledR}$, Ferring BV, Hoofddorp, The Netherlands) at $0.1 \mathrm{mg} /$ day s.c., starting on Cycle Day 21 (CD21) of the menstrual cycle preceding the actual stimulation cycle. After two weeks of the GnRH-agonist regimen, co-treatment with rFSH 225 IU/day s.c. (Puregon ${ }^{\circledR}$, MSD Haarlem, Haarlem, The Netherlands) was initiated. Patients receiving mild $\mathrm{COH}$ were treated with a fixed dose of $150 \mathrm{IU} /$ day $\mathrm{rFSH}$ s.c. (Puregon ${ }^{\circledR}$, MSD Haarlem, Haarlem, The Netherlands) from CD 5 onwards. As soon as the leading follicle reached a diameter of $14 \mathrm{~mm}$, a GnRH-antagonist (Orgalutran ${ }^{\circledR}$, MSD Haarlem, Haarlem, The Netherlands) was administered at $0.25 \mathrm{mg} /$ day s.c. To induce final oocyte maturation a single s.c. dose of 10,000 IE hCG (Pregnyl ${ }^{\circledR}$, MSD Haarlem, Haarlem, The Netherlands) was administered in both regimens as soon as the leading follicle reached a diameter of at least $18 \mathrm{~mm}$ and at least one additional follicle reached a diameter of $15 \mathrm{~mm}$ or more. Oocytes were retrieved 36 hours after hCG injection by transvaginal ultrasound-guided aspiration of follicles. Isolated oocytes were washed and transferred to a separate droplet of medium in order to monitor quality. On day 3 after oocyte pick up a maximum of two embryos was transferred. Pregnancy confirmation was assessed by a biochemical pregnancy test in urine 15 days after oocyte retrieval. Ongoing pregnancy is defined as positive heart action at 10 weeks, confirmed by ultrasound. Hereafter, couples return to their midwives for pregnancy follow-up.

\section{Sample Collection and Analysis}

Venous blood samples were drawn from each woman on $\mathrm{CD} 2$, i.e. the early follicular phase of the menstrual cycle proceeding the treatment cycle and on the day of hCG administration for the determination of serum folate, Red Blood Cell folate, pyridoxine, homocysteine, estradiol, FSH and $\mathrm{AMH}$. The methods used to determine the concerning biomarker concentrations have been extensively described elsewhere [20].

\section{Dietary Pattern Analysis}

All 195 food items from the FFQ data of all participants were classified into 22 food groups and adjusted for total energy intake, according to the residual energy adjusted method [22]. Therefore, only women with a completed FFQ could be included into these analyses, excluding 21 women. This was followed by Principle Components Analysis (PCA) applied on intake of the energy-adjusted food groups of women to construct overall dietary patterns by explaining the largest proportion of variation in the food group. Based on the scree-plot and the explained variance (eigen-value $>1.25$ ), the first three factors were considered for further dietary pattern analysis (see Supplementary table for the components of the three eligible dietary patterns). Each woman was assigned personal scores for the respective factors, calculated as the product of the food group value and its factor loadings summed across foods. According to the personal scores the group of women was divided into tertiles and classified as low, intermediate, or high adherence to the respective dietary pattern. The strength of adherence indicates the resemblance of the woman's diet compared with the respective dietary pattern identified by PCA.

Prior to baseline analyses, all continuous variables were log-transformed, obtaining a near normal 


\section{Women's Health Science Journal}

distribution of data suitable for parametric statistical testing. Measures of location and spread are depicted as Geometric Mean and Interquartile Range, respectively.

ANOVA was applied to compare the three tertiles of adherence. Proportions were compared using the Chisquare test. To allow for adjustment for confounders, multivariable linear and logistic regression methods were used to investigate the relation between dietary patterns, patient characteristics and endocrine responses after $\mathrm{COH}$. For the effect of dietary patterns on the $\mathrm{E}_{2}$ response to $\mathrm{COH}$, we considered as potential confounders baseline $\mathrm{AMH}, \mathrm{E}_{2}, \mathrm{FSH}$ and serum and RBC folate concentrations, number of follicles, total FSH dose and age, BMI and multivitamin supplement use (including folic acid supplement use only) of the woman. For the effect of diet on ongoing pregnancy chance we considered as potential confounders the number of transferred embryo's, age, BMI and multivitamin supplement use of the woman, treatment indication, fertilization rate, woman and partner smoking status, ethnicity and education. We applied a backward analysis, with the inclusion of interaction variables and squared variables. During the backward process a variable was included into the analysis if its p-value $<0.10$ and inclusion changed the effect estimate of the variable of interest $>10 \%$. All dependent variables used for the linear regression analyses were log-transformed for two reasons: to achieve satisfying adherence to normality and to achieve a log-level linear regression model, which allows for more intuitive interpretation of the $\beta$ coefficients. The interpretation of the $\beta$ is that $\beta \times 100$ equals the $\%$ change in the dependent variable for each 1-unit change in $\beta$. The relevant regression parameters are reported with their
$95 \%$ confidence intervals. Collinearity was assessed using the VIF statistic, a VIF of $\geq 4$ was considered to indicate collinearity. In the final model the highest observed VIF was 2.0. Statistical analysis was performed using SPSS 17.0 for Windows software (SPSS Inc, Chicago, IL).

\section{Results}

We identified three habitual dietary patterns (Table S1 for details) and further explored associations with $\mathrm{E}_{2}$ response using linear regression analysis. In contrast to the first two dietary patterns, the third dietary pattern significantly associates with the $\mathrm{E}_{2}$ response and was therefore further analyzed $(\mathrm{r}=-0.16 ; \mathrm{p}=0.02)$. This diet is characterized by high intake of fruits, whole grains, margarine, and low intake of refined grains and snacks; it explained $8.9 \%$ of variance found in dietary intake and is further named the habitual Healthy dietary pattern. To compare baseline characteristics of women over the range of scores of adherence to the habitual Healthy dietary pattern, we divided the cohort into three equally sized groups of adherence to the habitual Healthy dietary pattern, i.e. low, medium and high. Table 1 depicts baseline characteristics of women undergoing IVF/ICSI treatment. In the high adherence group, a larger proportion of women reported the use of a folic acid containing (multi)vitamin supplement. However, the exclusion of women who did not use a vitamin supplement from the forthcoming regression analyses did not alter the results (see further). Other baseline characteristics show a comparable distribution over the tertiles of adherence (Table 1).

\begin{tabular}{|c|c|c|c|}
\hline & $\begin{array}{c}\text { First dietary pattern (Variance } \\
\text { explained: 10.3\%) }\end{array}$ & $\begin{array}{c}\text { Second dietary pattern (Variance } \\
\text { explained: 9.5\%) }\end{array}$ & $\begin{array}{c}\text { Third (Healthy) dietary pattern } \\
\text { (Variance explained: 8.9\%) }\end{array}$ \\
\hline Alcohol &, 039 &, 009 &,- 009 \\
\hline Cereals &, 198 &, 559 &, 026 \\
\hline Butter &, 013 &, 666 &, 130 \\
\hline Dairy &,- 033 &,- 136 &, 098 \\
\hline Eggs &,- 081 &, 453 &, 125 \\
\hline Fish &, 633 &,- 012 &, 622 \\
\hline Fruits &, 357 &, 044 &, 161 \\
\hline Legumes &, 526 &, 090 &, 293 \\
\hline Margarine &,- 089 &,- 684 &,- 130 \\
\hline Mayonnaise &,- 021 &,- 186 &,- 008 \\
\hline Meat &,- 517 &,- 211 &, 019 \\
\hline Non-alcohol &,- 056 &,- 029 &, 148 \\
\hline Nuts &, 078 &, 079 &,- 579 \\
\hline Refined grains &, 393 &,- 092 &,- 111 \\
\hline Potato &,- 020 &, 060 & \\
\hline
\end{tabular}




\section{Women's Health Science Journal}

\begin{tabular}{|c|c|c|c|}
\hline Sauces &, 079 &,- 038 &,- 075 \\
\hline Snacks &,- 096 &,- 231 &,- 448 \\
\hline Soup &, 057 &,- 095 &, 031 \\
\hline Sugars &,- 190 &, 259 &,- 096 \\
\hline Vegetable oils &, 138 &, 208 &,- 189 \\
\hline Vegetables &, 683 &,- 073 &, 096 \\
\hline Whole grains &, 042 &,- 235 &, 705 \\
\hline
\end{tabular}

Table S1: Food group loadings for eligible habitual dietary patterns.

\begin{tabular}{|c|c|c|c|c|}
\hline & Low (n=68) & Medium (n=68) & High (n=67) & p \\
\hline Age (years) & $34.3(31.0-38.0)$ & $33.9(31.3-37.0)$ & $35.1(33.0-38.0)$ & 0.24 \\
\hline Body Mass Index (kg/m $\left.{ }^{2}\right)$ & $23.7(21.0-26.3)$ & $22.9(20.0-25.0)$ & $22.6(20.0-25.0)$ & 0.22 \\
\hline Ethnicity: & & & & 0.74 \\
\hline Dutch \% (n) & $65.6(42)$ & $72.7(48)$ & $72.1(44)$ & \\
\hline Non-Dutch European \% (n) & $9.4(6)$ & $10.6(7)$ & $11.5(7)$ & \\
\hline Non-European \% (n) & $25.0(16)$ & $16.7(11)$ & $16.4(11)$ & \\
\hline Education: & & & & 0.69 \\
\hline Low \% (n) & $13.4(9)$ & $20.6(14)$ & $13.4(9)$ & \\
\hline Intermediate \% (n) & $38.8(26)$ & $41.2(28)$ & $41.8(28)$ & \\
\hline High \% (n) & $47.8(32)$ & $38.2(26)$ & $44.8(30)$ & 0.39 \\
\hline Cause of subfertility: & & & & \\
\hline Male \% (n) & $30.9(21)$ & $35.3(24)$ & $46.3(31)$ & \\
\hline Female \% (n) & $29.4(20)$ & $22.1(15)$ & $14.9(10)$ & \\
\hline Combined \% (n) & $5.9(4)$ & $5.9(4)$ & $3.0(2)$ & \\
\hline Unknown \% (n) & $33.8(23)$ & $36.8(25)$ & $35.8(24)$ & \\
\hline Folic acid containing (multi)vitamin supplement use \% (n) & $80.9(55)$ & $83.8(57)$ & $94.0(63)$ & 0.024 \\
\hline Total FSH dose (IU) (median) IQR) & $1200(1050-1350)$ & $1200(1050-1500)$ & $1350(1200-1650)$ & 0.47 \\
\hline Stimulation protocol: & & & & 0.49 \\
\hline GnRH-agonist \% (n) & $7.4(5)$ & $13.2(9)$ & $9.0(6)$ & \\
\hline GnRH-antagonist \% (n) & $92.6(63)$ & $86.8(59)$ & $91.0(61)$ & \\
\hline IVF \% (n) & $71.6(48)$ & $60.3(41)$ & $70.6(48)$ & 0.63 \\
\hline Smokers \% (n) & $7.3(5)$ & $13.2(9)$ & $7.6(5)$ & 0.41 \\
\hline Duration of subfertility (months) & $33.1(24.0-50.0)$ & $36.3(27.3-51.0)$ & $35.2(25.3-51.0)$ & 0.83 \\
\hline
\end{tabular}

Table 1: Baseline characteristics of women undergoing controlled ovarian hyperstimulation, stratified for adherence to the habitual Healthy dietary pattern $(\mathrm{n}=203)$.

Variables are depicted: Geometric Mean (Interquartile Range), unless stated otherwise.

Table 2 shows biomarkers measured in serum prior to $\mathrm{COH}$ on $\mathrm{CD} 2$ and after stimulation treatment on the day of hCG administration. First, although not significant, baseline $\mathrm{E}_{2}$ and $\mathrm{AMH}$ concentrations are lower in the tertile of highest adherence (Table 2). In addition, serum concentrations of pyridoxine (PLP) and RBC folate concentrations are higher in the highest tertile (Table 2). After $\mathrm{COH}$, the differences are comparable with PLP and RBC folate concentrations being higher in the highest tertile of adherence. Furthermore, AMH and $E_{2}$ concentrations are lowest in the highest tertile (Table 2).
Albeit there was a statistically significant increase in $\mathrm{E}^{2}$ on the day of hCG, this increase was not statistically different between the three strata of adherence to the Healthy dietary pattern. However, when data were adjusted for total FSH dose, age, BMI, number of visualized follicles and baseline AMH concentrations, a non-linear (quadratic) association between adherence to the habitual Healthy dietary pattern and hCG-day $\mathrm{E}_{2}$ concentrations (r-model $=0.73 ; \beta_{\text {adherence }}=-0.11[-0.18$ - $0.04] ; \mathrm{p}<0.01$ and $\beta_{\text {adherence, squared }}=-0.054$ [-0.09 - -0.017$]$; $\mathrm{p}<0.01$ ) could be established. Thus, a one-point increase 


\section{Women's Health Science Journal}

in adherence to the habitual Healthy dietary pattern lowers hCG-day $\mathrm{E}_{2}$ concentrations by $16.4 \%$.

\begin{tabular}{|c|c|c|c|c|}
\hline & Low $(n=68)$ & Medium $(n=68)$ & High (n=67) & $\mathbf{p}$ \\
\hline Follicles (n) & $7(5-11)$ & $8(5-12)$ & $7(5-11)$ & 0.53 \\
\hline \multicolumn{5}{|l|}{ Baseline serum } \\
\hline Estradiol (pmol/L) & $143.6(109.5-185.8)$ & $146.1(115.0-185.8)$ & $134.0(108.0-167.0)$ & 0.5 \\
\hline AMH (ug/L) & $4.2(2.7-7.4)$ & $4.7(2.5-7.9)$ & $3.6(2.2-7.6)$ & 0.16 \\
\hline RBC folate $(\mathrm{nmol} / \mathrm{L})$ & $1,245(830-1,681)$ & $1,256(922-1,860)$ & $1,493(1,070-2,033)$ & 0.06 \\
\hline Folate (nmol/L) & $30.6(20.5-42.2)$ & $27.9(16.4-39.8)$ & $33.9(25.1-40.6)$ & 0.15 \\
\hline Homocysteine (umol/L) & $9.0(7.8-10.2)$ & $10.0(7.9-11.2)$ & $9.5(7.8-10.9)$ & 0.16 \\
\hline \multicolumn{5}{|l|}{ hCG-day serum } \\
\hline PLP (nmol/L) & $77.7(61.5-97.0)$ & $73.2(57.0-89.5)$ & $84.2(65.8-107.3)$ & 0.11 \\
\hline RBC folate (nmol/L) & $1,322(997-1,937)$ & $1,242(975-1,730)$ & $1,503(1,125-1,931)$ & 0.05 \\
\hline Folate $(\mathrm{nmol} / \mathrm{L})$ & $31.8(20.2-48.7)$ & $31.3(20.7-41.8)$ & $38.2(25.9-64.4)$ & 0.12 \\
\hline Homocysteine (umol/L) & $8.3(7.0-9.6)$ & $9.1(7.4-10.4)$ & $8.5(6.9-9.7)$ & 0.22 \\
\hline
\end{tabular}

Table 2: Biochemical markers of women undergoing controlled ovarian hyperstimulation, stratified for adherence to the habitual Healthy dietary pattern $(n=203)$.

Variables are depicted: Geometric Mean (Interquartile Range)

Table 3 shows the clinical reproductive outcome after IVF/ICSI treatment. There are no statistically significant differences between the groups. Although the fertilization rates appear different, this is likely an artifact of the fact that a binomial variable is created using a denominator and numerator with a skewed distribution. The number of suitable embryos is comparable. In the multivariable analyses, adjusted for the number of transferred embryo's, age of the woman, BMI of the woman, treatment indication, fertilization rate and woman smoking status, we show a significant association between adherence to the habitual Healthy dietary pattern and the subsequent chance of ongoing pregnancy $\left(\mathrm{OR}_{\mathrm{adj}}\right.$ : 1.61 [1.07 - 2.44]; $\left.\mathrm{p}=0.02\right)$.

\begin{tabular}{|c|c|c|c|c|}
\hline & Low (n=68) & Medium (n=68) & High (n=67) & p \\
\hline Retrieved oocytes (n) median (IQR) & $7(4-10)$ & $7(5-12)$ & $6(4-9)$ & 0.24 \\
\hline Fertilization rate \% & 65 & 51 & 50 & 0.28 \\
\hline Number of embryo's (n) median (IQR) & $4(2-6)$ & $3(2-6)$ & $3(1-6)$ & 0.74 \\
\hline Positive pregnancy test \% (n) & $23.5(16)$ & $25.0(17)$ & $35.8(24)$ & 0.24 \\
\hline Ongoing pregnancy \% (n) & $17.9(12)$ & $19.1(13)$ & $26.9(18)$ & 0.39 \\
\hline Embryo transfer & & & & 0.86 \\
\hline None \% (n) & $11.9(8)$ & $9.7(6)$ & $9.1(6)$ & \\
\hline Single \% (n) & $71.6(48)$ & $79.0(49)$ & $74.2(49)$ & \\
\hline Double \% (n) & $16.4(11)$ & $11.3(7)$ & $16.7(11)$ & \\
\hline
\end{tabular}

Table 3: Clinical outcome parameters after controlled ovarian hyperstimulation, stratified for adherence to the habitual Healthy dietary pattern $(n=203)$. 


\section{Women's Health Science Journal}

\section{Discussion}

This study shows that strong adherence to a periconceptional habitual Healthy dietary pattern characterized by high intake of fruit, whole grains and margarine and low intake of refined grains and snacks associates with lower $\mathrm{E}_{2}$ concentrations on the day of hCG and a higher chance of ongoing pregnancy after IVF/ICSI treatment.

The results from this study should be considered within the context of its strengths and limitations. The FOLFO study is a prospective periconception cohort designed to address the influence of nutrition and lifestyle on IVF/ICSI treatment outcome. In this study we used all participants of both the observational and randomized controlled trial, besides overweight status and patient preference there where no differences in inclusion criteria in either study, additionally, all analyses where adjusted for BMI and stimulation protocol. The habitual dietary patterns obtained using PCA from FFQ data result in sensible food-item based outcomes that are easily translated into patient care. However, it is important to consider the tendency of the individual to over represent socially desirable food habits and generally underestimate their overall food intake. Also, food habits are culturally determined, sometimes requiring an adapted FFQ for different ethnicities [23], in this study however, excluding non-western ethnicities did not alter the result. Furthermore, recall bias when completing FFQ is not an issue of concern because of the prospective design in which the outcome of the study was not known at the moment of the FFQ data collection. Even though PCA tends to emphasize sample specific factors; the high accordance between the various studies in the generated dietary patterns that are considered healthy and their associations with treatment outcome is a testament to the external validity of this method $[16,24]$.

Controlling the $\mathrm{E}_{2}$ response to $\mathrm{COH}$ using a modifiable factor, such as nutrition, could help to further personalize IVF/ICSI treatment and possibly increase the chance of pregnancy. In our study, however, the ovarian response itself was not a significant predictor of the chance of ongoing pregnancy. The role of high hCG-day $\mathrm{E}_{2}$ concentrations on clinical IVF/ICSI treatment outcome remains much debated, with an uncertain influence [6-9]. However, the effect of a high average number of transferred embryos $(\geq 3)$ in these studies is likely to dilute or distort any effects of the $E_{2}$ response on these clinical outcomes. In addition, in vitro studies clearly show that high $E_{2}$ concentrations associate with endometrial gene expression profiles that are considered not beneficial for implantation $[3,25]$. Possibly the influence of the ovarian response on IVF/ICSI treatment outcomes should not be solely due to the effects of $E_{2}$ on the endometrium, but also due to the influence of $\mathrm{COH}$ and the ovarian response (of which $\mathrm{E}_{2}$ is a marker) on oocyte and follicular developmental competency, by influencing follicular gene-expression profiles $[10,11]$, but also because the cytochrome P450 enzyme reaction cycles (used for steroid synthesis) results in a significant oxidative load [12] and thereby possibly oxidative stress.

In our study, the chance of ongoing pregnancy was highest in women with strong adherence to the Healthy dietary pattern, which is in accordance with a previous study in the same cohort [16]. Unfortunately, we were not able to study the influence on live birth, of ultimate relevance for couples undergoing IVF/ICSI treatment. The beneficial effects of reduced oxidative stress and folic acid supplement use on embryo and placental quality could lead to beneficial reproductive outcomes, possibly without affecting the ovarian response $[15,18,26]$. This is supported by the finding that nutrition also affects the chance of pregnancy in couples not undergoing IVF/ICSI treatment [24]. Therefore, improved pregnancy rates and a lower $E_{2}$ response can pose two separate effects of nutrition. Further studies need to address whether the association between nutrition, the ovarian response and improved pregnancy rates have a common causal pathway and if so; whether there is a cumulative effect of nutrition on IVF/ICSI treatment outcomes by affecting both embryo quality and the $\mathrm{E}_{2}$ response.

Several studies show beneficial effects of multivitamin supplement use including folic acid on IVF/ICSI treatment outcomes. In our study women with the highest adherence to the habitual Healthy dietary pattern were most likely to report multivitamin supplement use. This indeed raises the question whether a Healthy diet or multivitamin supplement use determines the outcomes? Nevertheless, including multivitamin supplement use in the analyses or excluding all nonusers did not influence the significant effects of nutrition on the ovarian response or chance of pregnancy. This suggests indeed that the observed associations are mediated through the habitual Healthy diet.

Earlier studies allow for speculation on possible mechanisms through which nutrition affects the ovarian response. Previously we showed that folic acid supplement use lowered the ovarian response to $\mathrm{COH}$ comparable to a diet high in fruit and whole grains 


\section{Women's Health Science Journal}

characterized by higher folate concentrations in the current study [17]. Additionally, Kanakkaparambil, et al. similarly showed mechanistic insight into an increased $\mathrm{E}_{2}$ response in ewes who received an opposite poor methyldeficient diet, through up regulation of the Follicle Stimulating Hormone-Receptor (FSHR) as a consequence of excessive oxidative stress [27]. Physiological up regulation of FSHR may also induce an anti-oxidant response through up regulation of glutathione $[28,29]$. Also, many polymorphisms in folate metabolism have been identified, which have been shown to influence ovarian metabolism [30]. However, since there is no known influence of these polymorphisms on nutritional habits, it is unlikely that these polymorphisms confound our findings (i.e. mendelian randomization). It is also interesting to note that at baseline women with high adherence to the habitual Healthy dietary pattern have lower AMH levels, a predictor for ovarian reserve and ovarian response. It would be of interest to confirm the hypothesis that a healthy dietary pattern would lead to a lower antral follicle count and thereby a lower ovarian response. Nevertheless, we suggest that the beneficial effects of the habitual Healthy dietary pattern could be mediated by the accompanied higher folate concentrations. The anti-oxidant capability of folate and the high anti-oxidant content of fruits propose a mechanism through which both can independently affect the $E_{2}$ response through reducing oxidative stress, notwithstanding the fact that a healthy dietary pattern is a prerequisite of adequate folate levels.

\section{Conclusion}

We show that strong adherence to a periconceptional habitual Healthy dietary pattern decreases the $E_{2}$ response to $\mathrm{COH}$, while increasing the subsequent chance of ongoing pregnancy after IVF/ICSI treatment. These findings support the rationale and necessity for initiation of the use of a healthy diet in women planning pregnancy, but also legitimize preconception counseling with regard to the influence of nutrition on IVF/ICSI treatment outcomes. Furthermore, this finding provides room for a more personalized approach of $\mathrm{COH}$ treatment. It is unclear whether the decreased $\mathrm{E}_{2}$ response or improved embryo quality through better nutrition is causal for the higher pregnancy rates, possibly it is both. In vitro research of granulosa cells could therefore help pinpoint underlying mechanisms. The anti-oxidant capacities of high fruit consumption may explain the effects on both the $E_{2}$ response and improved pregnancy rates and is in accordance with previous evidence.

\section{Acknowledgements}

The authors gratefully acknowledge the contribution of Dr. Jolanda Boxmeer and Professor Nick Macklon (Department of Obstetrics \& Gynecology, University of Southampton) to the FOLFO Study. We also thank the IVF team of the Division of Reproductive Medicine for the sample collection and the laboratory technicians and research assistants of the Laboratories of Clinical Chemistry and Endocrinology of the Erasmus MC, University Medical Centre in Rotterdam, The Netherlands, for the laboratory determinations.

\section{Contribution to Authorship}

JMT analyzed the data and wrote the manuscript. MV analyzed the data and provided analysis tools. JHdV provided essential materials and tools. JL provided reagents and analysis tools. JSEL was responsible for the clinical care of the patients and contributed to the writing of the manuscript. RPMST conceived the study, supervised all aspects and contributed to all versions of the manuscript.

\section{References}

1. Malizia BA, Hacker MR, Penzias AS (2009) Cumulative live-birth rates after In Vitro Fertilization. $\mathrm{N}$ Eng J Med 360(3): 236-243.

2. Fauser BCJM, Diedrich K, Devroey P (2008) Predictors of ovarian response: progress towards individualized treatment in ovulation induction and ovarian stimulation. Hum Reprod Update 14(1): 1-14.

3. Horcajadas JA, Díaz Gimeno P, Pellicer A, Simón C (2007) Uterine receptivity and the ramifications of ovarian stimulation on endometrial function. Semin Reprod Med 25(6): 454-460.

4. Bourgain C, Devroey P (2003) The endometrium in stimulated cycles for IVF. Hum Reprod Update 9(6): 515-522.

5. Koot YE, Macklon NS (2013) Embryo implantation: biology, evaluation, and enhancement. Curr Opin Obstet Gynecol 25(4): 274-279.

6. Simon C, Cano F, Valbuena D, Remohi J, Pellicer A (1995) Clinical evidence for a detrimental effect on uterine receptivity of high serum oestradiol concentrations in high and normal responder patients. Hum Reprod 10(9): 2432-2437. 


\section{Women's Health Science Journal}

7. Yu Ng EH, Yeung WSB, Yee Lan Lau E, So WWK, Ho PC (2000) High serum oestradiol concentrations in fresh IVF cycles do not impair implantation and pregnancy rates in subsequent frozen-thawed embryo transfer cycles. Hum Reprod 15(2): 250-255.

8. Peña JE, Chang PL, Chan LK, Zeitoun K, Thornton MH, et al. (2002) Supraphysiological estradiol levels do not affect oocyte and embryo quality in oocyte donation cycles. Hum Reprod 17(1): 83-87.

9. Papageorgiou T, Guibert J, Goffinet F, Patrat C, Fulla Y, et al. (2002) Percentile curves of serum estradiol levels during controlled ovarian stimulation in 905 cycles stimulated with recombinant FSH show that high estradiol is not detrimental to IVF outcome. Hum Reproduction 17(11): 2846-2850.

10. de los Santos MJ, Garcia Laez V, Beltran Torregrosa D, Horcajadas JA, Martinez Conejero JA, et al. (2012) Hormonal and molecular characterization of follicular fluid, cumulus cells and oocytes from pre-ovulatory follicles in stimulated and unstimulated cycles. Hum Reprod 27(6): 1596-1605.

11. Pellicer A (1997) Oestrogens and follicular and oocyte development. Hum Reprod Update 3(2): 9394.

12. Lewis D (2002) Oxidative stress: the role of cytochromes P450 in oxygen activation. J Chem Technol Biotechnol 77(10): 1095-1100.

13. Andersen AN, Witjes H, Gordon K, Mannaerts B (2011) Predictive factors of ovarian response and clinical outcome after IVF/ICSI following a $\mathrm{rFSH} / \mathrm{GnRH}$ antagonist protocol with or without oral contraceptive pre-treatment. Hum Reprod 26(12): 3413-3423.

14. van Disseldorp J, Lambalk CB, Kwee J, Looman CWN, Eijkemans MJC, et al. (2010) Comparison of inter- and intra-cycle variability of anti-Müllerian hormone and antral follicle counts. Hum Reprod 25(1): 221-227.

15. Boxmeer JC, Macklon NS, Lindemans J, Beckers NGM, Eijkemans MJC, et al. (2009) IVF outcomes are associated with biomarkers of the homocysteine pathway in monofollicular fluid. Hum Reprod 24(5): 1059-1066.

16. Vujkovic M, de Vries JH, Lindemans J, Macklon NS, van der Spek PJ, et al. (2010) The preconception
Mediterranean dietary pattern in couples undergoing in vitro fertilization/intracytoplasmic sperm injection treatment increases the chance of pregnancy. Fertil Steril 94(6): 2096-2101.

17. Twigt JM, Hammiche F, Sinclair KD, Beckers NG, Visser JA, et al. (2011) Preconception folic acid use modulates estradiol and follicular responses to ovarian stimulation. J Clin Endocrinol Metab 96(2): E322-9.

18. Steegers Theunissen R, Twigt J, Pestinger V, Sinclair K (2013) The periconceptional period, reproduction and long-term health of offspring: the importance of one-carbon metabolism. Hum Reprod Update 19(6): 640-655.

19. Anderson K, Norman R, Middleton P (2010) Preconception lifestyle advice for people with subfertility. Cochrane Database Syst Rev (4): CD008189.

20. Boxmeer JC, Steegers Theunissen RPM, Lindemans J, Wildhagen MF, Martini E, et al. (2008) Homocysteine metabolism in the pre-ovulatory follicle during ovarian stimulation. Hum Reprod 23(11): 2570-2576.

21. Siebelink E, Geelen A, de Vries JHM (2011) Selfreported energy intake by FFQ compared with actual energy intake to maintain body weight in 516 adults. Br J Nutr 106(2): 274-281.

22. Willett W, Howe G, Kushi LH (1997) Adjustment for total energy intake in epidemiologic studies. Am J Clin Nutr 65(4): 1220S-1228S.

23. Thompson F, Subar A (2001) Dietary assessment methodology. (Edn.), In: Coulston A, et al. (Eds.), Nutrition in the prevention and treatment of disease. San Diego: Academic Press: 3-30.

24. Toledo E, Lopez del Burgo C, Ruiz Zambrana A, Donazar M, Navarro-Blasco Í, et al. (2011) Dietary patterns and difficulty conceiving: a nested casecontrol study. Fertil Steril 96(5): 1149-1153.

25. Liu Y, Kodithuwakku SP, Ng PY, Chai J, Ng EHY, et al. (2010) Excessive ovarian stimulation up-regulates the Wnt-signaling molecule DKK1 in human endometrium and may affect implantation: an in vitro co-culture study. Hum Reprod 25(2): 479-490. 


\section{Women's Health Science Journal}

26. Di Simone N, Riccardi P, Maggiano N, Piacentani A, D'Asta M, et al. (2004) Effect of folic acid on homocysteine-induced trophoblast apoptosis. Mol Hum Reprod 10(9): 665-669.

27. Kanakkaparambil R, Singh R, Li D, Webb R, Sinclair KD (2009) B-Vitamin and homocysteine status determines ovarian response to gonadotropin treatment in sheep. Biol Reprod 80(4): 743-752.

28. Tilly JL, Tilly KI (1995) Inhibitors of oxidative stress mimic the ability of follicle- stimulating hormone to suppress apoptosis in cultured rat ovarian follicles. Endocrinology 136(1): 242-252.
29. Tsai Turton M, Luderer U (2006) Opposing effects of glutathione depletion and follicle-stimulating hormone on reactive oxygen species and apoptosis in cultured preovulatory rat follicles. Endocrinology 147(3): 1224-1236.

30. Altmäe S, Stavreus Evers A, Ruiz JR, Laanpere M, Syvänen T, et al. (2010) Variations in folate pathway genes are associated with unexplained female infertility. Fertil Steril 94(1): 130-137.

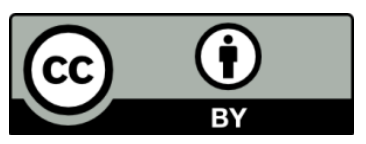

